\title{
Algoritmo de Alocação Dinâmica de Largura de Faixa para Redes Sem Fio
}

\author{
Eduardo Martinelli Galvão de Queiroz, Helvécio M. de Almeida Neto e Amílcar Careli César
}

\begin{abstract}
Resumo-Um controle de admissão de chamadas (CAC) para as redes celulares da terceira geração (3G) é proposto neste artigo. Em uma rede celular, o controle de admissão de chamadas exerce papel fundamental no desempenho geral do sistema, pois toma a decisão de aceitar ou não uma chamada de acordo com critérios estabelecidos. $O$ estudo leva em conta os diferentes tipos de chamadas presentes em uma rede celular e os resultados mostram melhorias de até $19 \%$ na probabilidade de bloqueio de novas chamadas.
\end{abstract}

Palavras-Chave-redes celulares de terceira geração (3G), controle de admissão de chamadas (CAC), alocação de banda.

Abstract-A call admission control (CAC) for third generation (3G) cellular networks is proposed in this paper. The CAC deals with the important task of accepting or not a new call according to criteria, including bandwidth. The analysis considers different classes of traffic such as variable bit rate and constant bit rate. Results show improvement up to $19 \%$ in the blocking probability of new calls keeping handoff dropping probability within suitable range.

Keywords - third generation (3G) cellular networks, call admission control, bandwidth management.

\section{INTRODUÇÃo}

O perfil de uso da telefonia móvel vem apresentando mudanças durante os últimos anos. Com o avanço da infraestrutura de comunicação celular como a de terceira geração $(3 \mathrm{G})$, os serviços de faixa larga apresentam rápido crescimento entre os usuários. Estes serviços incluem diferentes tipos de tráfego, como voz, vídeo e dados [1]. Os usuários destes serviços esperam uma qualidade de serviço (QoS) que depende de cada aplicação e também do serviço contratado da operadora. Desta maneira, a rede de telefonia celular deve garantir a qualidade de serviço de cada usuário e, ao mesmo tempo, maximizar o número de usuários que podem ser atendidos [2], utilizando de forma ótima os recursos.

A cobertura de uma rede celular é dividida em áreas menores, chamadas células, servidas cada uma por uma estação rádio base [3]. Atualmente, técnicas como GSM, TDMA e CDMA são utilizadas para o acesso aos recursos do sistema e técnicas como a EDGE (evolution of GSM for existing spectrum) chegam a oferecer serviços de até 384 kbps [4]. Várias abordagens indicam que largura de faixa de freqüências (banda) de cada estação rádio base pode ser tratada independentemente da técnica de acesso utilizada [2], [5][12]. No sistema de uma rede celular, o controle de admissão de chamadas (CAC - call admission control) exerce papel

Eduardo Martinelli Galvão de Queiroz, equeiroz@sel.eesc.usp.br, Helvécio M. de Almeida Neto, asah@terra.com.br, Amílcar Careli César, amilcar@sel.eesc.usp.br, Universidade de São Paulo, EESC, Depto. Eng. Elétrica Av. Trabalhador São-carlense, 400, 13566-590 São Carlos, SP, Brasil.

Este trabalho foi parcialmente financiado por CAPES e CNPq. fundamental no desempenho da rede de comunicação móvel e o seu funcionamento leva em conta vários fatores de decisão, como utilização de banda e QoS [13]. Este controle toma a decisão de aceitar ou não uma chamada tendo como base o tipo de serviço requisitado e suas características específicas. Essas decisões precisam ser tomadas em tempo real e, desta maneira, o algoritmo de admissão de chamadas precisa ser passível de uma implementação nos recursos de computação limitados das estações de rádio base [13].

Em uma rede celular, a geração de uma chamada pode ocorrer em qualquer célula do sistema. Esta chamada, por sua vez, pode ficar sob a responsabilidade desta única célula ou então pode passar para a responsabilidade de uma célula vizinha, devido à movimentação do usuário. O procedimento de mudança de célula é o handoff [1].

Neste sistema, a probabilidade de bloqueio de novas chamadas (PBNC) e a probabilidade de bloqueio de chamadas handoff $(\mathrm{PBCH})$ são dois parâmetros que demonstram o desempenho da rede. A PBNC estabelece a probabilidade de uma nova chamada ser bloqueada em uma célula, enquanto que a PBCH estabelece a probabilidade de uma chamada em curso ser finalizada quando deveria ocorrer a mudança para outra célula. O término de uma chamada devido ao handoff é considerado mais problemático para o usuário do que o bloqueio de uma nova chamada e, sendo assim, é desejável que a rede dê maior prioridade às chamadas handoff [2].

Um outro parâmetro importante é a utilização de banda, ou seja, como uma rede de telefonia celular consegue gerenciar sua capacidade de banda. O estudo do controle de admissão de chamadas foi realizado em vários trabalhos, como em [5]-[19], que se diferenciam pela maneira como realizam o gerenciamento de banda.

A contribuição do artigo é a proposição de um esquema de empréstimo de banda que melhora o desempenho dos CACs relatados na literatura. O CAC gerencia as novas chamadas geradas no sistema e também as chamadas handoff.

$\mathrm{O}$ artigo está dividido da seguinte maneira. Na próxima Seção são apresentados os trabalhos relacionados com o estudo realizado; na Seção III são expostos a problemática da alocação de recursos em redes celulares, os parâmetros relacionados e o esquema de CAC proposto. Na Seção IV são apresentados os resultados obtidos. Finalmente, na Seção $\mathrm{V}$, é realizada a conclusão do artigo.

\section{TRABAlhos Relacionados}

$\mathrm{Na}$ literatura há vários artigos que tratam do controle de admissão de chamadas. Alguns métodos envolvem programação linear [6], [16], redes neurais [13] e métodos heurísticos [2], [7]-[9]. 
Em [2] é proposto um controle de admissão de chamadas que trata seis diferentes tipos de tráfego com prioridades diferentes e divide a banda utilizada por cada tipo de chamada em $N$ níveis. O algoritmo realiza o empréstimo de banda de chamadas em andamento nas células para atingir níveis mínimos de banda $\left(B_{\min }\right)$ estabelecidos para novas chamadas e chamadas handoff. O algoritmo repõe a banda emprestada de cada chamada assim que o tráfego de chamadas em cada célula permitir.

Em [9] um controle de admissão de chamadas estabelece prioridades para chamadas em andamento e para novas chamadas. A banda necessária para cada chamada, que é dividida em $N$ níveis, especifica um nível mínimo $B_{\min }$ e um nível máximo $B_{\max }$. A diferença $\left(B_{\max }-B_{\min }\right)$ é o decréscimo máximo de banda que um certo tipo de chamada pode suportar. $\mathrm{O}$ trabalho também realiza o empréstimo de banda de chamadas em andamento para alocar novas chamadas e não estuda especificamente as chamadas handoff, que segundo os autores, podem ser representadas por chamadas de alta prioridade.

Seguindo a mesma proposta de divisão de banda, [7] estabelece um algoritmo para o controle de admissão de chamadas que tenta primeiramente alocar a banda desejada para cada chamada, que por sua vez é discretizada em $N$ níveis, tendo um nível máximo $M$ e nível mínimo $m$. Quando não há banda suficiente na célula, o esquema realiza o empréstimo temporário de banda de algumas chamadas em andamento na célula.

Em [8] a discretização de banda em $N$ níveis também é realizada, assim como o empréstimo de banda de chamadas em andamento para a alocação de novas chamadas e chamadas handoff. A distribuição de banda entre as chamadas em andamento é realizada baseada no esquema Max-Min, mas não utiliza nenhum esquema de adaptação do tamanho da banda reservada às chamadas handoff.

Em [10] um esquema de empréstimo de banda é realizado para a alocação de chamadas quando a banda disponível na célula não é suficiente para atender o nível de banda máximo de uma chamada. $\mathrm{O}$ algoritmo possui um critério pelo qual as novas chamadas originadas em uma célula são bloqueadas se o número de chamadas correntes for maior ou igual a um determinado valor.

Em [12] é implementado um algoritmo que busca automaticamente o número ideal de canais que são alocados para as chamadas handoff em cada estação base. Esta busca é realizada com o monitoramento da probabilidade de bloqueio de chamadas handoff e com a introdução de um valor limiar (threshold) para este parâmetro.

Em [15] é proposto um esquema baseado na característica apresentada pelo tráfego web (WWW). Como este tipo de tráfego apresenta períodos onde não há transferência de dados, algumas conexões são agrupadas e compartilham a mesma banda utilizando uma política round robin.

Neste artigo propomos um controle de admissão de chamadas (CAC) que inclui o empréstimo de banda de chamadas correntes nas células e também da banda reservada às chamadas handoff conforme certos parâmetros estabelecidos. O controle trata os seis tipos de chamadas consideradas com prioridades diferentes no que diz respeito à "granularidade" de cada tipo de tráfego. Os resultados mostram melhorias de até $19 \%$ na probabilidade de bloqueio de novas chamadas em relação ao esquema de empréstimo de banda proposto por [7].

\section{Controle de Admiss Ão de Chamadas Proposto}

\section{A. O Problema de Alocação de Recursos em Redes Celulares}

$\mathrm{O}$ aumento da demanda por serviços multimídia em comunicações móveis vem crescendo rapidamente nas últimas duas décadas. Um dos desafios da rede de telefonia celular é oferecer os serviços requisitados pelos usuários e ao mesmo tempo lidar com a escassez de banda do sistema [10].

Neste sentido, a principal questão reside em como alocar recursos que melhorem o desempenho geral de rede, como a probabilidade de bloqueio de novas chamadas e de chamadas handoff. A alocação de recursos nas redes celulares pode ser entendida por meio da associação de atividades que requerem recursos do sistema [18], que decide se cada solicitação será atendida ou não. Neste caso, adota-se que os recursos na rede celular são distribuídos em $m$ diferentes portas (recursos) para serem alocados em $n$ atividades. A atividade $j$ corresponde à requisição de uma célula para um recurso. Pode-se tomar $\Re j(t)$ como o total de recursos que a atividade $j$ requer no tempo $t$ no sistema e que varia dinamicamente com o tempo. A demanda de uma atividade $j$ na célula $i$ e no tempo $t$ para um determinado recurso pode ser representado por $x_{i, j}(t)$. Assim temos que a demanda total de uma atividade $j$ pode ser representada pela seguinte expressão

$$
\sum_{i=1}^{m} x_{i, j}(t)=\Re j(t), 1 \leq j \leq n
$$

Estabelecendo $N_{i}$ como o total de recursos de uma célula $i$ e considerando que o total de requisições para um recurso na célula $i$ é menor do que o total de recursos do sistema, $N$, então

$$
\sum_{i=1}^{m} \sum_{j=1}^{n} x_{i, j}(t) \leq N \text { e } N \prec \sum_{i=1}^{m} N_{i}, \text { para } 1 \leq i \leq m
$$

Da mesma forma, o total de recursos utilizados na célula $i$ será

$$
r_{i}(t)=\sum_{j=1}^{n} x_{i, j}(t), 1 \leq i \leq m
$$

O objetivo de um controle de admissão de chamadas é a maximização do uso dos recursos do sistema e ao mesmo tempo promover bom desempenho da rede, o que se caracteriza por uma baixa probabilidade de bloqueio de novas chamadas e de chamadas handoff. Estes dois parâmetros podem ser obtidos pelo número de chamadas bloqueadas ou que sofreram interrupção em relação à quantidade total de cada uma e são calculados por

$$
P B_{N}=\frac{X_{n b}}{N_{n}}
$$




$$
P B_{H}=\frac{X_{h b}}{N_{h}}
$$

nas quais $P B_{N}$ é a probabilidade de bloqueio de novas chamadas, $P B_{H}$ é a probabilidade de bloqueio de chamadas handoff, $X_{n b}$ representa o total de novas chamadas bloqueadas, $N_{n}$ representa o total de novas chamadas, $X_{h b}$ representa o total de chamadas handoff bloqueadas e $N_{h}$ representa o total de chamadas handoff.

\section{B. Parâmetros}

Cada classe de serviço (Tabela I) contém um nível máximo $\left(N_{\max }\right)$ e um nível mínimo $\left(N_{\min }\right)$ de banda e, assim, a tolerância de perda $(T P)$ possível pode ser expressa como

$$
T P=N_{\max }-N_{\min }
$$

Os intervalos de banda $I B_{i}$ são determinados entre $N_{\min }$ e $N_{\max }$. Estes intervalos são definidos por um parâmetro, $\beta_{i}$, que determina os níveis possíveis de conexão para cada classe $i$. A equação seguinte mostra a definição dos intervalos.

$$
I B_{i}=\frac{T P}{\beta_{i}}
$$

O sistema de empréstimo de banda pode ser entendido como um sistema justo de adaptação dos níveis de conexão conforme o tráfego de cada célula. Um outro parâmetro, chamado adaptabilidade (AD), pode ser definido por [7]

$$
A D=\frac{T P}{N_{\max }}
$$

De acordo com (8), quanto maior for a adaptabilidade da conexão, ou seja, quanto maior sua tolerância de perda em relação a um mesmo nível máximo de conexão $\left(N_{\max }\right)$, menor será a probabilidade de bloqueio no caso de handoff, já que seu intervalo pelo qual os níveis de conexão são implementados é maior.

Supondo que em uma célula do sistema uma conexão $C$ da classe $i$ esteja operando em um nível $L$ de conexão, o seu nível de conexão $(N C)$ será

$$
N C=N_{\max }-L \times \frac{T P}{\beta_{i}}
$$

Para a mesma conexão $C$ a sua razão de perda $(R P)$ pode ser calculada por

$$
R P=\frac{L \times \frac{T P}{\beta_{i}}}{N_{\max }}
$$

Pela manipulação direta de (10) tem-se

$$
R P=\frac{L}{\beta_{i}} \times \frac{N_{\max }-N_{\min }}{N_{\max }}=\frac{L}{\beta_{i}} \times A D
$$

Desta maneira, para uma determinada célula o fator $L / \beta_{i}$ é constante e assim uma conexão da classe $i$ vai se estabelecer em um nível de conexão proporcional à sua adaptabilidade.

\section{Esquema proposto}

O esquema considera a existência de seis diferentes classes de chamadas, como sugerido em [2], [7]-[9], cada uma contendo um nível máximo e um nível mínimo de taxa de transmissão. As diferentes classes de chamadas estão representadas na Tabela I. A primeira coluna especifica a classe da chamada e a última mostra a taxa de geração das chamadas (em \%) em relação ao total de chamadas geradas. A coluna do meio especifica a média de duração de cada classe.

TABELA I

Classes de Chamadas

\begin{tabular}{|c|c|c|c|c|c|}
\hline & $B_{\min } B_{\max }$ & Média(s) & Uso & Tipo & $\%$ \\
\hline 1 & $30-30 \mathrm{kbps}$ & 180 & Voz & CBR & $40 \%$ \\
\hline 2 & $256-256 \mathrm{kbps}$ & 300 & Video I & CBR & $15 \%$ \\
\hline 3 & $1-6 \mathrm{Mbps}$ & 600 & Video II & VBR & $10 \%$ \\
\hline 4 & $1-20 \mathrm{kbps}$ & 30 & E-mail & VBR & $20 \%$ \\
\hline 5 & $64-512 \mathrm{kbps}$ & 180 & Download & VBR & $10 \%$ \\
\hline 6 & $1-10 \mathrm{Mbps}$ & 120 & Arquivo & VBR & $5 \%$ \\
\hline
\end{tabular}

Dentre os seis tipos de tráfego considerados, quatro deles são do tipo VBR (variable bit rate) e dois são CBR (constant bit rate), justamente as classes que não permitem variação em suas taxas de conexão.

As chamadas que pertencem às classes que permitem níveis diferentes de taxas de conexão são tratadas conforme sua tolerância à perda de banda. Desta maneira, cada tipo de tráfego VBR tem um nível mínimo aceitável e também uma tolerância diferente de perda de banda, devido aos diferentes valores de $\beta$, determinando assim as prioridades de cada chamada, conforme pode ser visualizado pela Figura 1. Portanto, quanto maior o valor de $\beta$, maior o número de níveis possíveis de conexão (maior "granularidade") e maior a prioridade da classe. $\mathrm{Na}$ literatura, o valor de $\beta$ varia de acordo com a abordagem [9], [10].

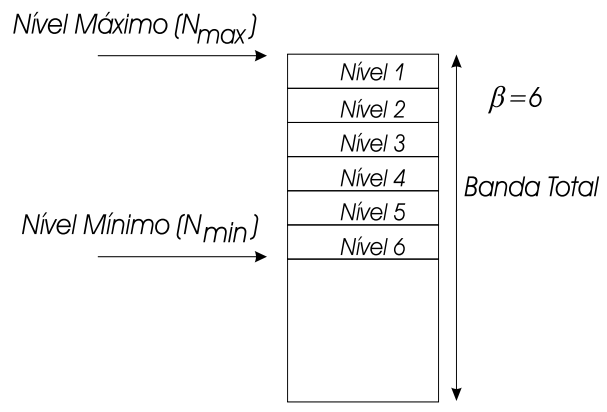

Fig. 1. Esquema de Divisão de Níveis de Conexão.

Assim, o tráfego da classe 3, por possuir uma "granularidade" maior do que o de classe 6, tem uma prioridade maior. Desta maneira, terá um número maior de níveis nos quais sua conexão poderá ser estabelecida, conforme pode ser visualizado pela Figura 2.

O controle de admissão de chamadas proposto opera com os níveis de conexão aceitáveis para cada classe de chamada e reserva para as chamadas do tipo handoff uma banda de $20 \%$ da capacidade total $(6 \mathrm{Mbps})$ de cada estação rádio 


\begin{tabular}{|c|c|c|c|}
\hline$\beta=6$ & $\beta=5$ & $\beta=4$ & $\beta=3$ \\
\hline Nível 1 & Nível 1 & Nível 1 & Nível 1 \\
\hline Nível 2 & Nivel 2 & Nível 2 & \\
\hline Nível 3 & Nível 3 & Nível 3 & Nivel 2 \\
\hline Nível 4 & N'vel 4 & Nível 4 & Nível 3 \\
\hline Nivel 5 & Nivel 5 & & \\
\hline Nível 6 & & & \\
\hline$N_{\min }$ & $N_{\min }$ & $N_{\min }$ & $N_{\min }$ \\
\hline lasse 3 & Classe 4 & Classe 5 & Classe 6 \\
\hline
\end{tabular}

Fig. 2. Níveis de Conexão das Classes de Tráfego.

base, que é adotada com o valor de $30 \mathrm{Mbps}$, assim como em [2], [5]-[9]. Os níveis de conexão são as possíveis taxas que um determinado tipo de tráfego pode estabelecer. $\mathrm{O}$ uso da banda reservada será feito quando a banda disponível da célula não for suficiente para acomodar chamadas do tipo handoff. Para cada nova solicitação de conexão gerada, o CAC proposto verifica a possibilidade de alocação do nível máximo de banda requisitado $\left(N_{\max }\right)$. No caso de banda insuficiente no sistema, é realizada uma verificação em ordem decrescente nos níveis de conexão permitidos para a chamada, até o nível mínimo $\left(N_{\min }\right)$. Se a banda disponível não for suficiente para a alocação de nenhum dos níveis da solicitação de conexão, é realizado um empréstimo de banda de chamadas que estão em andamento na célula. $O$ procedimento verifica as chamadas em ordem crescente de prioridade e em ordem decrescente de tempo de permanência na célula e diminui em uma unidade o nível de conexão presente nas conexões até que a banda acumulada pelo empréstimo seja igual ou maior do que o nível mínimo $\left(N_{\min }\right)$ requisitado pela solicitação de conexão. Cada chamada terá em seu tempo de permanência em uma célula apenas uma mudança do nível de conexão.

Neste artigo propomos o seguinte esquema. O sistema mantém em constante atualização dois parâmetros, $P B_{N}$ e $P B_{H}$, que armazenam a expectativa de probabilidade de bloqueio de novas chamadas e de chamadas handoff, respectivamente, para cada célula.

Quando há o empréstimo de banda, o sistema verifica essas duas variáveis. No caso de $P B_{N}$ estar acima de um certo limiar $\left(T_{N}\right)$ e $P B_{H}$ abaixo de outro $\left(T_{H}\right)$ e a variação da probabilidade de bloqueio de handoff $\left(\triangle P B_{H}\right)$ for negativa, (ou seja, se a probabilidade diminuiu em relação à última computação) a banda considerada para a alocação de uma nova chamada contará com o aumento da mesma magnitude da banda reservada para as chamadas handoff.

Com este tipo de abordagem, o controle de admissão de chamadas apenas utiliza a banda reservada às chamadas handoff para as novas chamadas no caso dos dois parâmetros utilizados não estarem sendo violados. Caso o esquema não consiga atingir o nível mínimo de banda $\left(N_{m i n}\right)$, então a chamada é bloqueada.

Para as chamadas handoff, os procedimentos são os mesmos, com exceção do monitoramento das variáveis $P B_{H}$ e $P B_{N}$.
Para cada chamada handoff, o controle de admissão verifica a possibilidade de alocar o nível máximo de banda para as classes de tráfego. Caso isso não seja possível devido à banda insuficiente disponível para a nova célula, o procedimento de empréstimo de banda de chamadas em andamento é iniciado da mesma maneira que é realizado para as novas chamadas, porém a chamada será alocada apenas se a banda disponível for suficiente para acomodar o nível médio de conexão $\left(N_{m e d}\right)$. Esta abordagem tenta minimizar a queda abrupta de taxa de conexão na ocorrência de handoff. O seu fluxograma está na Figura 3.

Quando uma chamada deixa uma célula, a banda ocupada pela conexão será adicionada à banda disponível da célula se a banda reservada para as chamadas handoff estiver completa ou, caso contrário, será adicionada à banda reservada. Após este procedimento, a banda ocupada é utilizada para o aumento do nível de conexão de chamadas que foram utilizadas para o empréstimo de banda.

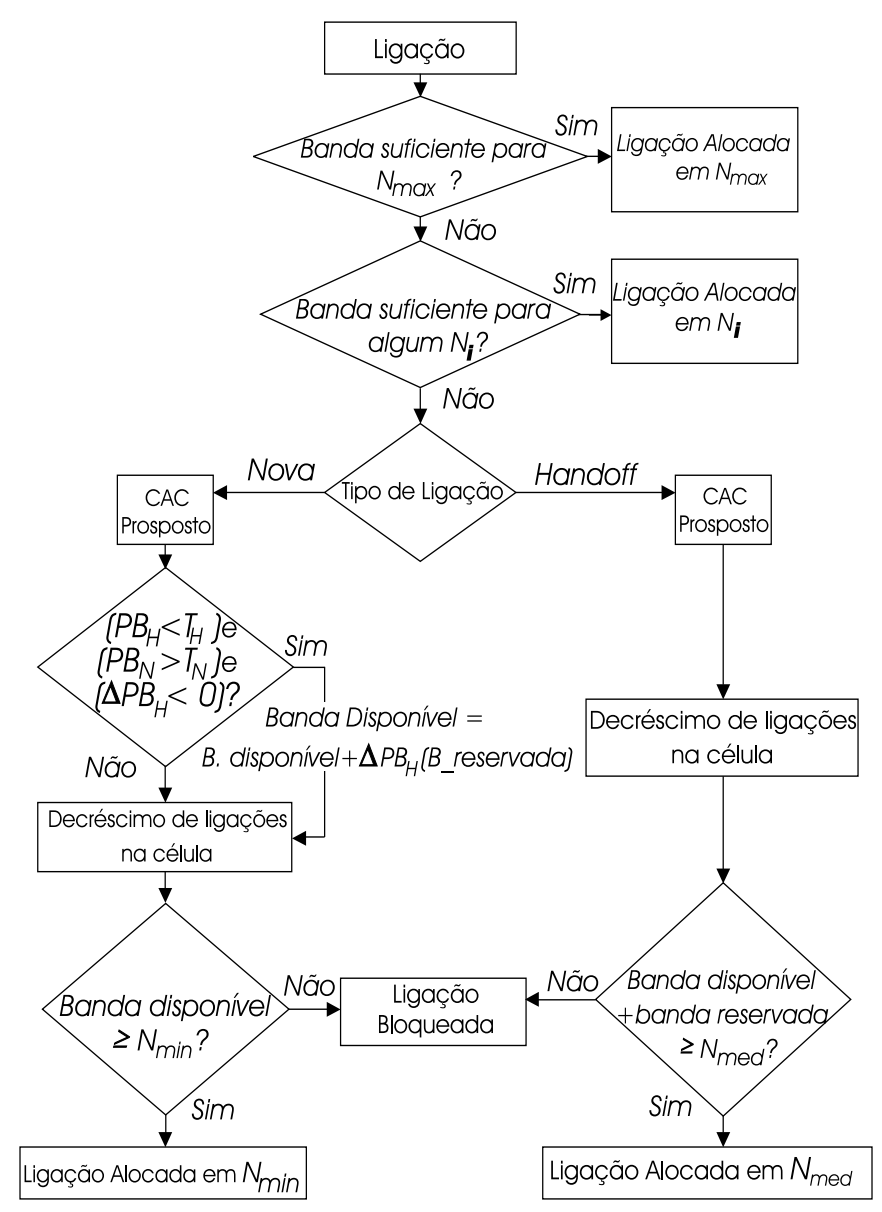

Fig. 3. Fluxograma do CAC Proposto.

\section{Simulação}

A simulação discreta de eventos da rede celular foi implementada em linguagem ANSI C e conta com 100 células hexagonais. O sistema simulado tem como foco a alocação de recursos em termos de banda disponível em cada estação rádio base, que tem uma capacidade de $30 \mathrm{Mbps}$. A geração de 
novas chamadas ocorre seguindo uma distribuição de Poisson, tendo como média o número de chamadas originadas por segundo $(\lambda)$. Cada chamada gerada tem uma probabilidade $P_{h}$ de sofrer até $N_{H(i)}$ handoffs. $i$ é a classe da chamada e o tempo de permanência em cada célula é igual para todas as células participantes da chamada.

A divisão das classes de chamadas segundo a Tabela I apresentada torna a geração das chamadas mais realista, já que o uso de uma rede celular é caracterizado principalmente por chamadas de voz. Este tipo de divisão foi encontrado em apenas um trabalho relacionado, [2], mas os percentuais de cada classe de chamada não foram divulgados.

\section{Resultados}

Os resultados são comparados com diferentes valores de $T_{N}$, que variam de $0,05 \lambda$ a $0,80 \lambda$, na qual $\lambda$ é a taxa de chegada das chamadas nas células. A comparação é feita com as configurações exibidas na Tabela II. O valor de $T_{H}$ foi definido como 0,02 e $P_{h}$ como 0,75 . Cada ponto dos gráficos corresponde à média aritmética de três simulações realizadas. Os intervalos de confiança não são mostrados nos gráficos porque seus valores são muito pequenos (cerca de 0,0002).

TABELA II

CONFIGURAÇÕES

\begin{tabular}{|c|c|c|}
\hline & Configuração & $T_{N}$ \\
\hline A & CAC sem empréstimo de banda & - \\
\hline B & CAC sem esquema proposto & - \\
\hline C & Adaptação proposta & $0,05 \lambda$ \\
\hline D & Adaptação proposta & $0,15 \lambda$ \\
\hline E & Adaptação proposta & $0,45 \lambda$ \\
\hline F & Adaptação proposta & $0,80 \lambda$ \\
\hline
\end{tabular}

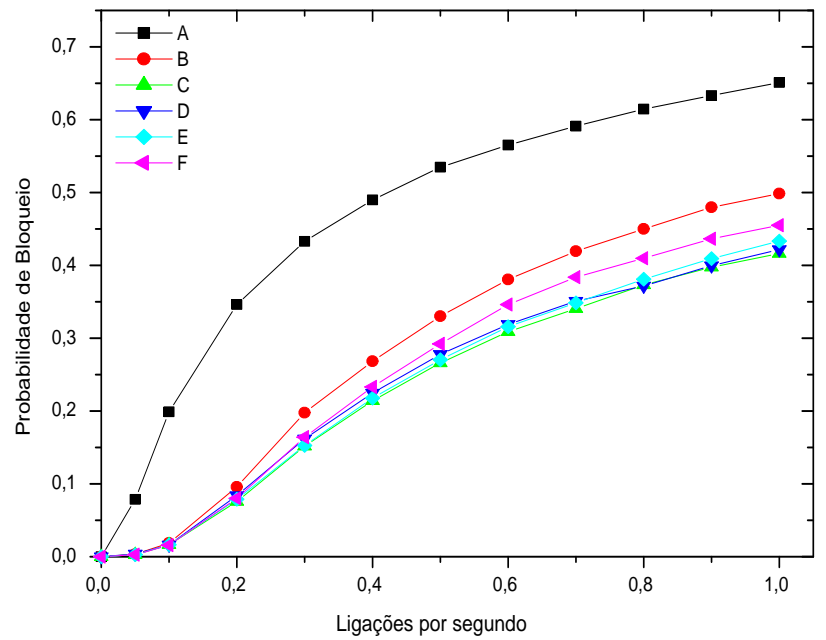

Fig. 4. Probabilidade de Bloqueio de Novas Chamadas (Ver Tabela III).

A Figura 4 mostra a probabilidade de bloqueio de novas chamadas para os diversos tipos de CAC estudados (A e
TABELA III

Comparação de Valores de Probabilidade entre os Esquemas (NOVAS CHAMADAS)

\begin{tabular}{|c|c|c|c|c|c|}
\hline$\lambda$ & $\begin{array}{c}\text { Prob. } \\
\text { CAC B }\end{array}$ & $\begin{array}{c}\text { Prob. } \\
T_{N}=0,05 \lambda\end{array}$ & Dif. & $\begin{array}{c}\text { Prob. } \\
T_{N}=0,15 \lambda\end{array}$ & Dif. \\
\hline 0,1 & 0,0187 & 0,0164 & -12 & 0,0164 & -12 \\
\hline 0,5 & 0,3300 & 0,2660 & -19 & 0,2704 & -18 \\
\hline 1,0 & 0,4985 & 0,4162 & -16 & 0,4333 & -13 \\
\hline
\end{tabular}

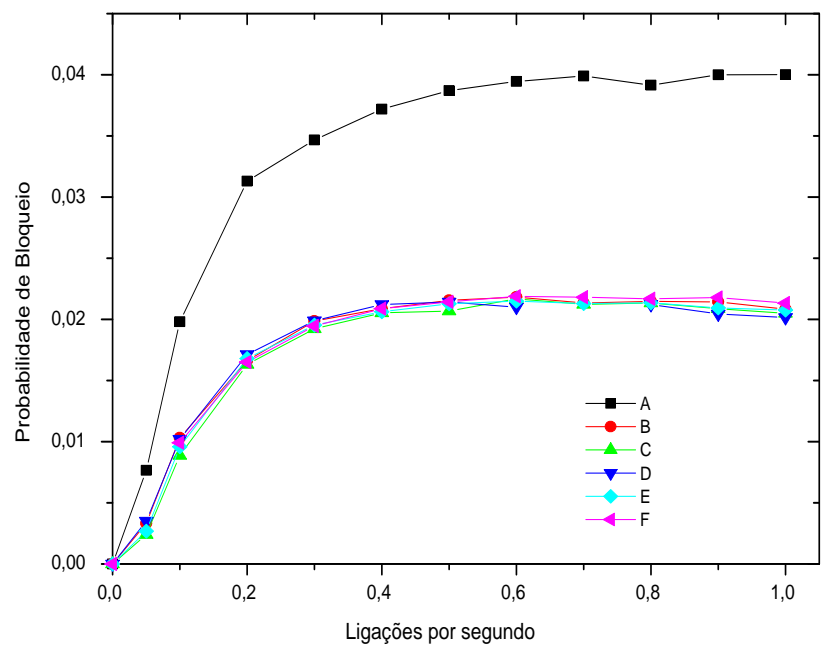

Fig. 5. Probabilidade de Bloqueio de Chamadas Handoff (Ver Tabela IV).

B) e pelo CAC proposto. O CAC A não adota o esquema de empréstimo de banda, enquanto que o CAC B realiza o empréstimo de banda sem a adaptação da banda reservada ao handoff. Nota-se que a adaptação de banda proposta melhora o desempenho do sistema conforme uma diminuição do limiar $T_{N}$. Este comportamento se deve ao fato de que quanto menor $T_{N}$, maior é o número de vezes que ocorrerá a adição de parte da banda reservada para chamadas handoff para a banda disponível para novas chamadas. Para efeito de análise, a Tabela III mostra valores da probabilidade de bloqueio de novas chamadas para três valores de $\lambda: 0,1,0,5$ e 1,0 . Ocorre uma melhora significativa de até $19 \%$ na probabilidade de bloqueio de novas chamadas quando utilizado o esquema proposto neste trabalho.

No caso da probabilidade de bloqueio de chamadas hand-

TABELA IV

Comparação de VAlores de Probabilidade entre os Esquemas (CHAMADAS handoff)

\begin{tabular}{|c|c|c|c|c|c|}
\hline$\lambda$ & $\begin{array}{c}\text { Prob. } \\
\text { CAC B }\end{array}$ & $\begin{array}{c}\text { Prob. } \\
T_{N}=0,05 \lambda\end{array}$ & Dif. & $\begin{array}{c}\text { Prob. } \\
T_{N}=0,15 \lambda\end{array}$ & Dif. \\
\hline 0,1 & 0,0103 & 0,0088 & -14 & 0,01015 & -2 \\
\hline 0,5 & 0,0215 & 0,0206 & -4 & 0,0214 & $-0,5$ \\
\hline 1,0 & 0,0208 & 0,0204 & -2 & 0,0201 & -3 \\
\hline
\end{tabular}




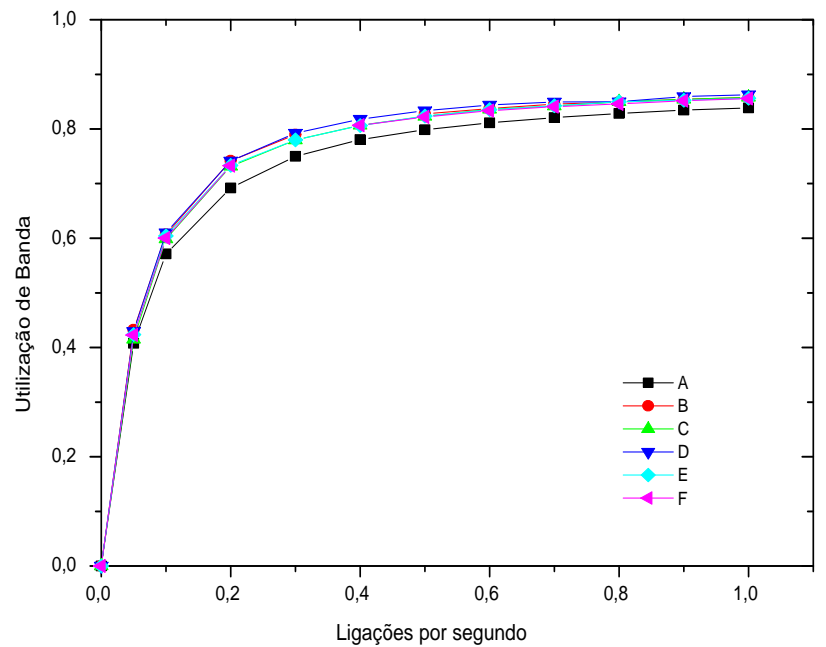

Fig. 6. Utilização de Banda (Ver Tabela V).

off não há uma clara diferenciação entre os esquemas de adaptação de banda estudados, conforme mostra a Figura 5. Apenas o esquema no qual não há adaptação diferencia-se e apresenta uma probabilidade de bloqueio maior. Pela Tabela IV é possível perceber as pequenas diferenças entre os valores apresentados pelos esquemas.

$\mathrm{O}$ esquema proposto privilegia as novas chamadas, pois utiliza a banda reservada às chamadas handoff, mas não influi negativamente na probabilidade de bloqueio de chamadas handoff, pois um outro limiar, $T_{H}$, é utilizado e esta banda é alocada apenas se também satisfaz este limiar.

TABELA V

Comparação de Valores de Probabilidade entre os Esquemas (UTILIZAÇÃO DE BANDA)

\begin{tabular}{|c|c|c|c|c|c|}
\hline$\lambda$ & $\begin{array}{c}\text { Prob. } \\
\text { CAC B }\end{array}$ & $\begin{array}{c}\text { Prob. } \\
T_{N}=0,05 \lambda\end{array}$ & Dif. $\%$ & $\begin{array}{c}\text { Prob. } \\
T_{N}=0,15 \lambda\end{array}$ & Dif.\% \\
\hline 0,1 & 0,6069 & 0,5985 & -1 & 0,6100 & $+0,5$ \\
\hline 0,5 & 0,8275 & 0,8230 & $-0,5$ & 0,8334 & $+0,7$ \\
\hline 1,0 & 0,8576 & 0,8580 & $+0,05$ & 0,8626 & $+0,5$ \\
\hline
\end{tabular}

Um outro parâmetro é a utilização da banda, mostrado na Figura 6. A melhora na utilização de banda quando é usado o esquema proposto ocorre com certos valores de $T_{N}$ e é resultado do melhor aproveitamento da banda reservada às chamadas handoff. A Tabela $\mathrm{V}$ exibe alguns valores para a utilização de banda e mostra que a relativa queda no aproveitamento de banda para alguns cenários não é significativamente grande.

\section{Conclusões}

Neste trabalho foi proposto um controle de admissão de chamadas (CAC) que possui um mecanismo de empréstimo de banda de chamadas em andamento nas células para o atendimento de novas chamadas geradas. $\mathrm{O}$ esquema agrega um mecanismo que determina o tamanho da banda reservada às chamadas handoff conforme os valores de alguns parâmetros que são computados para cada nova alocação.

Os resultados mostram uma melhoria significativa de até $19 \%$ num determinado cenário para a probabilidade de bloqueio de novas chamadas e de até $14 \%$ na probabilidade de bloqueio de chamadas handoff num cenário específico.

\section{REFERÊNCIAS}

[1] S. Faruque, "Cellular Systems Engineering", Artech House, 1996

[2] M. Sanabani, S. Shamala, M. Othman e J. Desa, "Adaptive Call Admission Control for Prioritized Adaptive services in Wireless/Mobile Multimedia Cellular Networks", International Journal of Computer Science and Network Security, pp.114-214, 2006.

[3] A. S. Tanembaum, "Redes de Computadores", Editora Campus, 2003

[4] T. Halonen, J. Melero, J. R. Garcia, "GSM, GPRS and EDGE Performance: Evolution Toward 3G/UMTS", Halsted Press, Nova Yorque, NY, 2002

[5] C. Oliveira, J. Kim, e T. Sud,. "Quality-of-Service Guarantee in HighSpeed Multimedia Wireless Networks". Technical Report, University of California, Irvine, CA, pp. 95-41, 1995.

[6] Koo-Min Ahn, Sehun Kim, "Optimal bandwidth allocation for bandwidth adaptation in wireless multimedia networks", Computers and Operations Research, Volume 30, nro.13, pp. 1917-1929, Novembro, 2003.

[7] M. El-Kadi, S. Olariu, e H. Abdel-Wahab, "A rate-based borrowing scheme for QoS provisioning in multimedia wireless networks", IEEE Trans. Parallel Distrib. Syst., Volume13, nro.2, pp.156-166, Jan. 2002.

[8] Malla, A., El-Kadi, M., Olariu, S., e Todorova, P., "A Fair Resource Allocation Protocol for Multimedia Wireless Networks". IEEE Trans. Parallel Distrib. Syst. 14, pp. 63-71, Janeiro, 2003.

[9] Mokhtar Aboelazel, Ayman Elnaggar e Maan Musleh, "A Priority Based Call Admission Control Protocol with Call Degradation for Cellular Networks", Proceedings of the 1st International Symposium on Wireless Communication Systems ISWC, Mauritius, pp. 20-22, Setembro, 2004.

[10] N. Nasser e H. S. Hassanein, "Combined Admission Control Algorithm and Bandwidth Adaptation Algorithm in Multimedia Cellular Networks with QoS Provisioning”, IEEE Canadian Conference on Electrical and Computer Engineering, Maio, 2004.

[11] Tewari, Maneesh e Jamadagni, HS, "A New Call Admission Control scheme for Real-time traffic in Wireless Networks". In Proceedings IEEE TENCON, Bangalore, Índia, pp. 1585-1589, 2003.

[12] Y. Zhang e D. Liu, "An adaptive algorithm for call admission control in wireless networks", in: Proc. IEEE Globecom, San Antonio, Texas, pp. 3628-3632, 2001

[13] Q. Ren, e G. Ramamurthy, "A real-time dynamic connection admission controller based on traffic modeling, measurement, and fuzzy logic control". IEEE Journal on Select. Areas Comm., Volume 18, pp. 268282, Fevereiro, 2000.

[14] Ghaderi, Majid e Boutaba, Raouf, "Call admission control in mobile cellular networks: a comprehensive survey", Wireless Communications \& Mobile Computing, pp. 69-93, Fevereiro, 2006.

[15] K. Jun, S. Kang, "Call Admission Control for Next Generation Cellular Networks Using on Demand Round Robin Bandwidth Sharing”, Lecture Notes in Computer Science, Volume 3421, pp. 543 - 550, Janeiro, 2005.

[16] Lima, Marcos Antonio Cardoso de, "Alocação de recursos e roteamento de tráfego em telecomunicações por meio de algoritmo genético: rede óptica WDM e rede de comunicação móvel celular", Tese de Doutorado, Escola de Engenharia de São Carlos - Universidade de São Paulo, 2005.

[17] Nidal Nasser e Hossam Hassanein, "Optimized Bandwidth Allocation with Fairness and Service Differentiation in Multimedia Wireless Networks", International Journal of Wireless Communications and Mobile Computing, Wiley, 2006.

[18] Raiyn, Jamal, "Development and Comparison Between Resource Allocation Strategies Based on Intelligent Scheme and Traditional Distributed Dynamic Resource Allocation Strategies in Mobile Communication Networks", Wireless Personal Communications: An International Journal, Volume 40, pp. 495 - 509, Março, 2007.

[19] Y.C. Kim, D.E. Lee, Y.S. Kim e B. Mukherjee, "Dynamic channel reservation based on mobility in wireless ATM networks", IEEE Communications Magazine, pp. 47-51, 1999. 